\title{
Design and Testing of a Vertical Scroll Pump Efficiency
} Jeerawat Pokanit, ${ }^{1 a}$ and Assist. Prof. Pollakrit Kritmaitree, $2 \mathrm{~b}$

\author{
${ }^{1}$ Mechanical Engineering Department, Faculty of Engineering, Kasetsart University, Thailand \\ ${ }^{2}$ Mechanical Engineering Department, Faculty of Engineering, Kasetsart University, Thailand \\ a pokanitjeer54@gmail.com , ${ }^{\mathrm{b}}$ fengpck@ku.ac.th
}

Keywords: scroll pump, vertical scroll pump, efficiency of pump, pump testing.

Abstract. This research aim designs and tests a vertical scroll pump. The mathematical models of the curves fixed and the orbiting scrolls were used in designing and constructing a vertical scroll pump and the principles of dynamic of machinery was applied to analysis the centrifugal force of a vertical scroll pump. The testing facilities were design and constructed under the British Standard (BS EN 143343) and the Japanese Industrial Standard (JIS B 8301). The characteristics of a vertical scroll pump, if the pressure increases, the flow rate of a vertical scroll pump will change a little at the constant speed. The relationship the efficiency of a vertical scroll pump and the pressure increase in the linear equation manner, in contrast, if the flow rate increases, the efficiency of a vertical scroll pump will reduce in the linear equation manner. For reducing of the centrifugal force on the main shaft can be able to increase the speed of a scroll pump. As the result, the scroll pump's the maximum of total efficiency is $27.75 \%$ at flow rate is $42.53 \mathrm{~L} / \mathrm{min}$ and pressure is $18.55 \mathrm{~m}$. The speed of a vertical scroll pump is $1500 \mathrm{rpm}$.

\section{Introduction}

The scroll pump is arranged in a kind of a positive displacement pump. The ideal characteristic of a positive displacement pump is the flow rate is constant and the pressure is changed by the friction loss in the system at the constant speed. The first research and development for the scroll pump were used for cooling the reactor, at that time the research has studied the principle of the compressor used water pump. [1] Since 2010, the scroll pump was developed, but there was low efficiency because of the scroll pump has leaked by wearing of the orbiting scroll and the fix scroll. [2] After that the scroll pump was developed to be more durable changing a material from alumminum to hardened steel. As the result that the scroll pump does not wear on the orbiting scroll and fixed scroll, so a scroll pump dives at high speed by the maximum of speed is $1100 \mathrm{rpm}$.[3] In the past the scroll pump was only a horizontal pump. This research designs and builts a scroll pump in a vertical pump, it is called a Vertical scroll pump. First type has the main components, an orbiting scroll, a fixed scroll and a main shaft as shown in Fig 1.

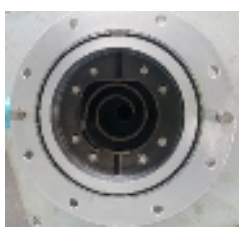

(a) A fixed scroll

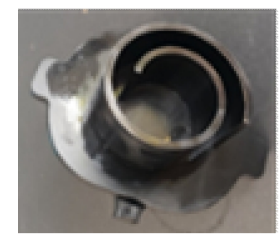

(b) An orbiting scroll

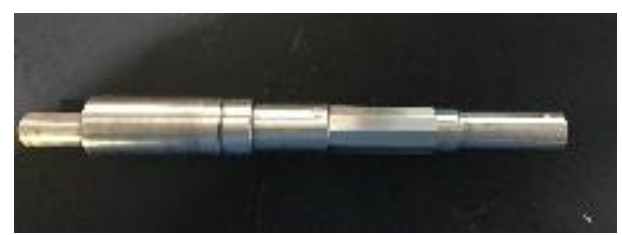

(c) A main shaft

Fig. 1: The main components of a vertical scroll pump

A vertical scroll pump works as same as the horizontal scroll pump that an orbiting is a moving part create a pressure inside a volumetric of scroll pump, the flow rate is increased by the speed of a scroll pump. 


\section{Centrifugal force analysis :}

The rotation of a orbiting scroll does not rotate on the center line of mass of a main shaft be the cause of the centrifugal force or the shaking force. If a scroll pump decreases this force, is increasing a speed of a scroll pump. The centrifugal force equation is (1)

$$
F=m \omega^{2} r
$$

When

$$
\begin{aligned}
& F=\text { Centrifugal force }[\mathrm{N} .] \\
& m=\text { Mass }[\mathrm{Kg}] \\
& r=\text { Radius of rotation }[\mathrm{m} .] \\
& \omega=\text { Angular Velocity }[\mathrm{Rad} / \mathrm{s} .]
\end{aligned}
$$

From analysis, the centrifugal force on main shaft of the horizontal scroll pump and a vertical scroll pump are as show in Fig.2 and Fig.3

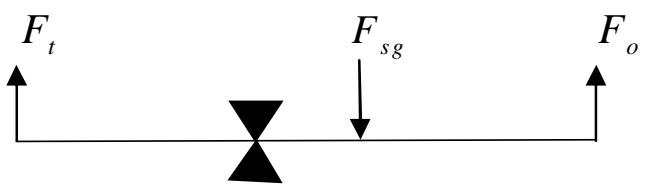

Bearing

Fig. 2: The centrifugal force on main shaft of the horizontal pump

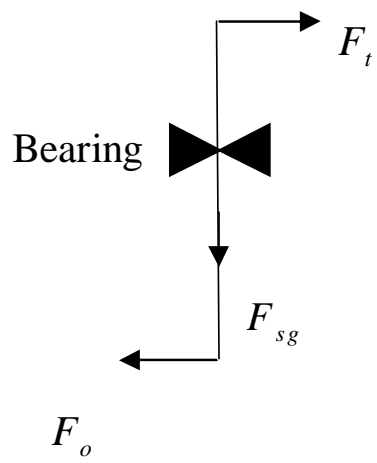

Fig. 3: The centrifugal force on main shaft of the vertical pump

When

$F_{t}=$ Centrifugal force on the main shaft $[\mathrm{N}$.

$F_{s g}=$ Centrifugal force by main shaft on the main shaft $[\mathrm{N}$.]

$F_{o}=$ Centrifugal force by orbiting scroll on the main shaft $[\mathrm{N}$.]

From Fig 1. and Fig 2. the centrifugal force on main shaft between the horizontal scroll pump and the vertical scroll pump is very different. A horizontal scroll pump includes the centrifugal force from orbiting scroll and gravity force of a main shaft but a vertical scroll pump include the centrifugal force of a orbiting scroll because the gravity force of a main shaft is on the center line of mass so the gravity force of a main shaft does not effect to a mian shaft. A vertical scroll pump is designed by this principle for increasing the speed of the scroll pump. 


\section{Algebraic spiral scroll equation :}

The orbiting scroll and the fixed scroll are designed by an algebraic spiral scroll equation. The algebraic spiral scroll equation is calculated as a coordinate by equation of inner curve and outer curve [1] as shown in Eq. 2 to Eq. 9

Fixed scroll

Inner curve

$$
\begin{aligned}
& x=\alpha(\cos \lambda+(\lambda+\pi-\beta) \sin \lambda) \\
& y=\alpha(\sin \lambda-(\lambda+\pi-\beta) \sin \lambda)
\end{aligned}
$$

When $2 \pi \leq \lambda \leq 5 \pi$

Outer curve

$$
\begin{aligned}
& x=\alpha(\cos \lambda+(\lambda-\pi) \sin \lambda) \\
& y=\alpha(\sin \lambda-(\lambda-\pi) \cos \lambda)
\end{aligned}
$$

When $2 \pi \leq \lambda \leq 5 \pi$

Orbiting scroll

Inner curve

$$
\begin{aligned}
& x=\alpha(\cos \lambda+(\lambda-\beta) \sin \lambda)+\gamma \cos \theta \\
& y=\alpha(\sin \lambda-(\lambda-\beta) \sin \lambda)+\gamma \cos \theta
\end{aligned}
$$

When $3 \pi \leq \lambda \leq 6 \pi$

Outer curve

$$
\begin{aligned}
& x=\alpha(\cos \lambda+\lambda \sin \lambda)+\gamma \cos \theta \\
& y=\alpha(\sin \lambda-\lambda \cos \lambda)+\gamma \sin \theta
\end{aligned}
$$

When $3 \pi \leq \lambda \leq 6 \pi$

For this research the fixed scroll and orbiting scroll were designed a scroll coefficient $(\alpha)$ value is $1.75 \mathrm{~mm}$. and a discrepancy of starting roll angle $(\beta)$ is $0.3 \pi$ radian and a crank Angle $(\theta)$ is 0 to $2 \pi$ radian as shown in Fig.4

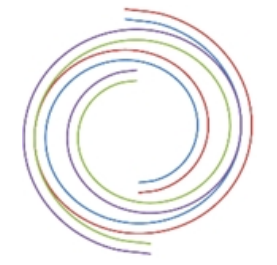

$\theta=0$ radian or $2 \pi$ radian

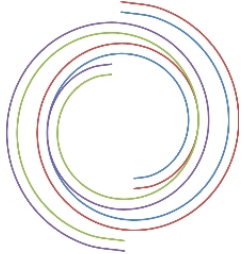

$\theta=\pi$ radian

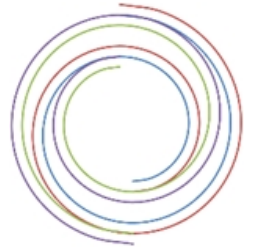

$\theta=0.5 \pi$ radian

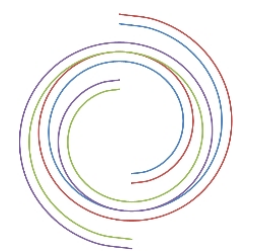

$\theta=1.5 \pi$ radian

Fig. 4: The spiral curves of the scroll pump is $\alpha=1.75 \mathrm{~mm} . \beta=0.3 \pi$ radian 


\section{Methodology of the experiment :}

At the present, a vertical scroll pump does not have a standard for testing. So, the testing of a vertical scroll pump will use British Standard (BS EN 143343) [4] and the Japanese Industrial Standard (JIS B 8301) [5] for reference. By measuring the power input of a vertical scroll pump will use the power consumption of electric motor, without using the value of a brake horsepower. Because we want to know the value of a total efficiency of a vertical scroll pump complete set. The experiment of a vertical scroll pump as shown in Fig. 5

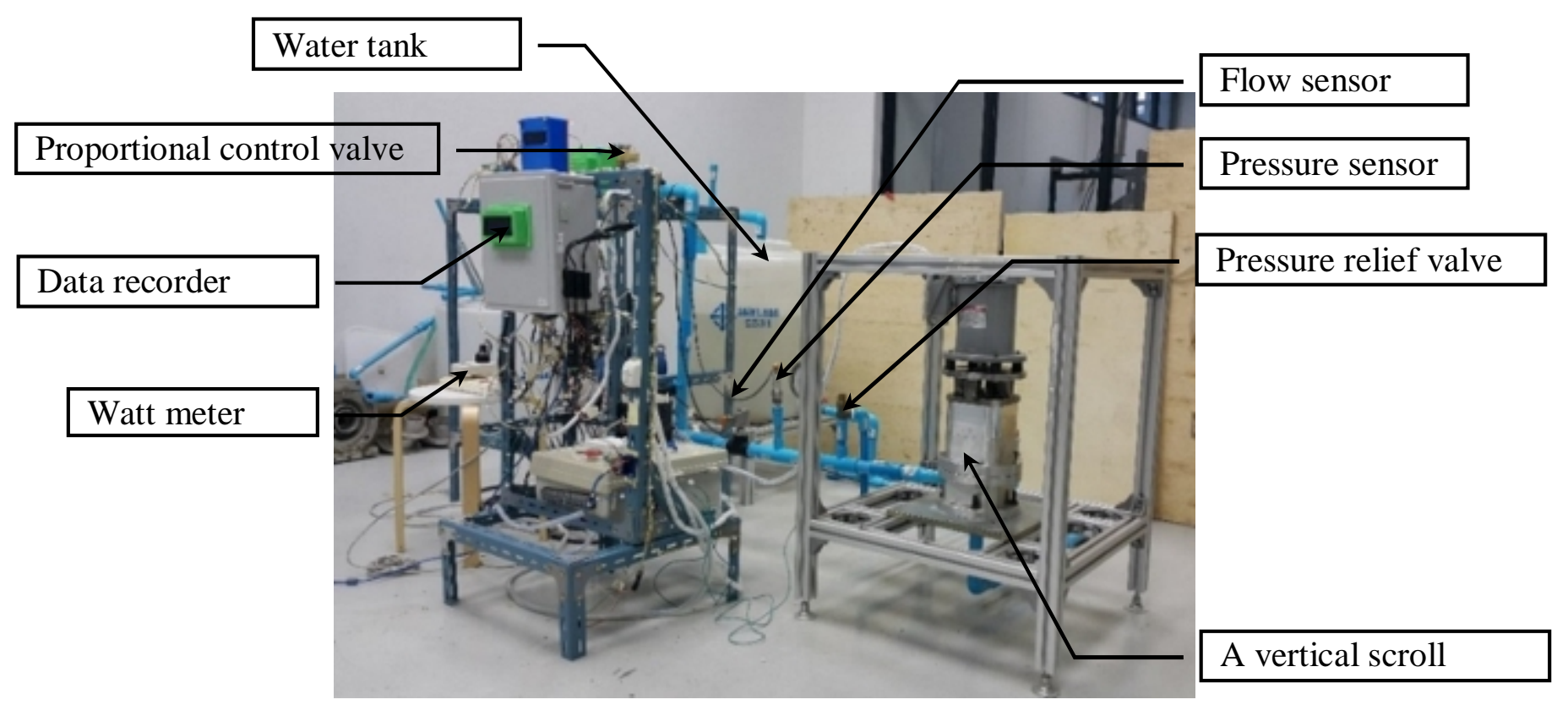

Fig. 5: The experimental of a vertical scroll pump

The efficiency of a vertical scroll pump is calculated by

$$
\begin{aligned}
& \eta(\%)=\frac{\text { Power }_{\text {output }}}{\text { Power }_{\text {input }}} \times 100 \\
& \text { Power }_{\text {output }}=\sigma g Q H_{P} \\
& \text { Power }_{\text {input }}=W_{e}
\end{aligned}
$$

When

$$
\begin{aligned}
& \eta=\text { The efficiency of the scroll pump } \\
& H_{P}=\text { Total the pressure }(\mathrm{m} .) \\
& \sigma=\text { The density of water }\left(\mathrm{Kg} / \mathrm{m}^{3}\right) \\
& Q=\text { The flow rate }(\mathrm{L} / \mathrm{min}) \\
& W_{e}=\text { The electrical power of motor (Watt) }
\end{aligned}
$$

\section{Results and Discussion :}

From the experiment, the relationship between the pressure of a vertical scroll pump and the flow rate of a vertical scroll pump are the linear manner as shown in Fig. 6, the maximum speed of a vertical scroll pump is $1500 \mathrm{rpm}$, the maximum flow rate is $48.90 \mathrm{~L} / \mathrm{min}$ and the maximum pressure is 18.55 $\mathrm{m}$. and the maximum efficiency is $27.75 \%$ at the speed is $1500 \mathrm{rpm}$. At the constant speed when the pressure increase by the loss of piping system and the flow rate will decrease by leakage between a fix scroll and a orbiting scroll, it is 4 to $5 \mathrm{~L} / \mathrm{min}$ at all the speed of testing. The relationship between the 
efficiency and the pressure as shown in Fig. 7. For the reasons already mentioned if a vertical scroll pump increases the flow rate, a vertical scroll pump decrease the efficiency. The relationship between the efficiency and the flow rate as shown in Fig. 8.

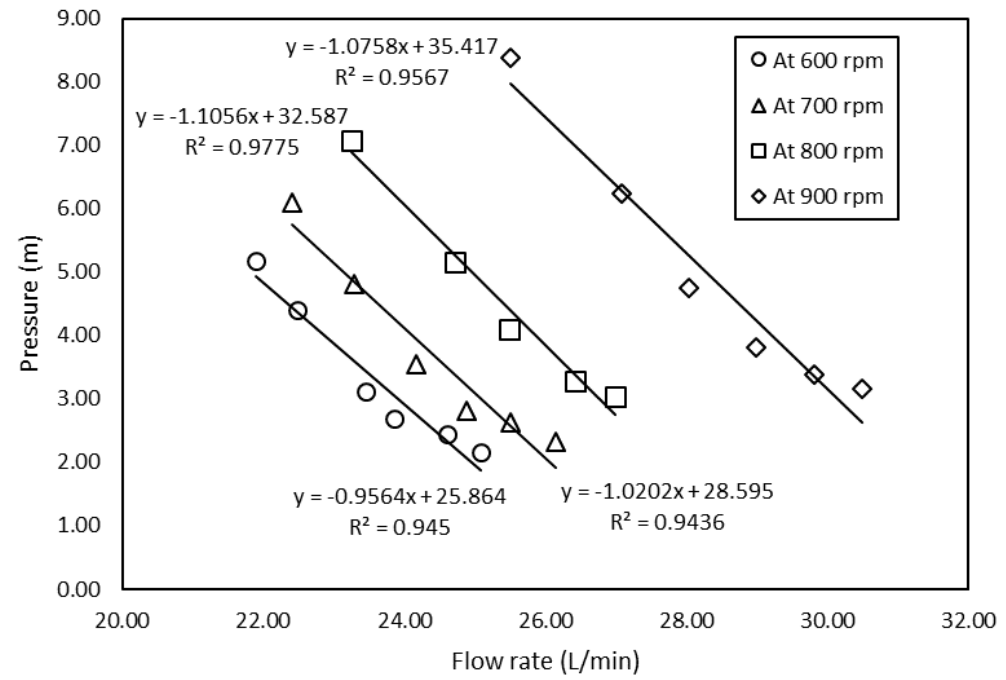

(a) The relationship between flow rate and pressure at 600 to $900 \mathrm{rpm}$.

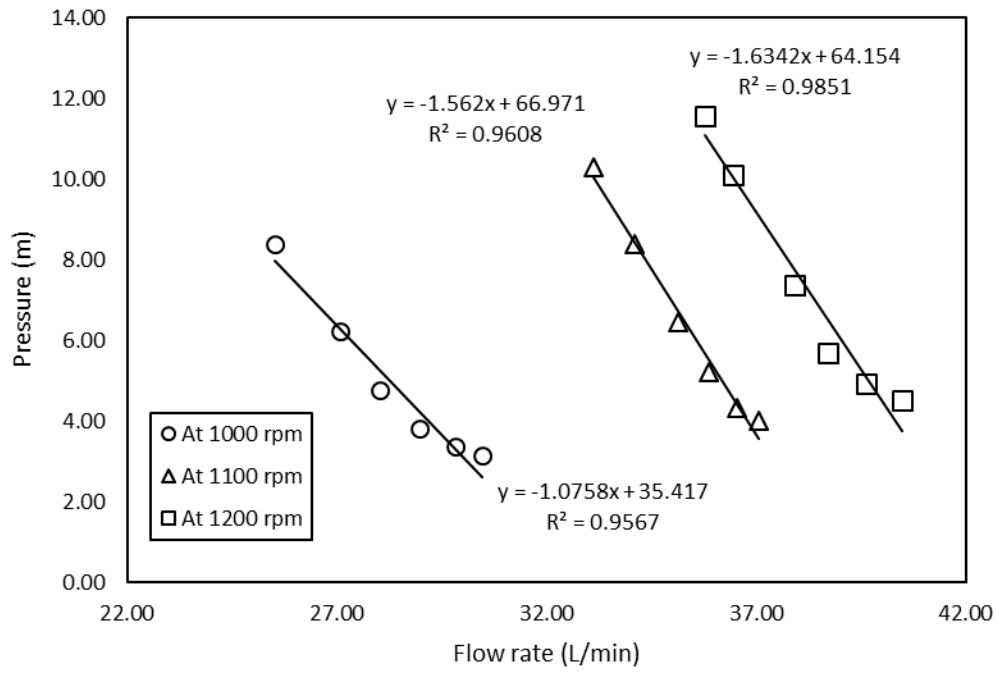

(b) The relationship between flow rate and pressure at 1000 to $1200 \mathrm{rpm}$.

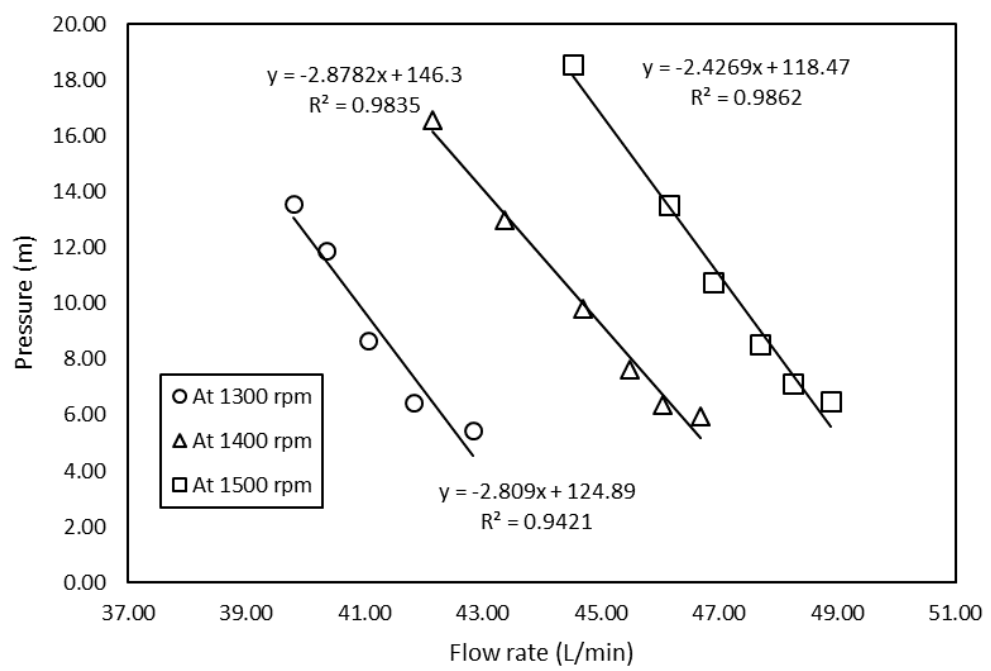

(c) The relationship between flow rate and pressure at 1300 to $1500 \mathrm{rpm}$.

Fig. 6: The relationship between flow rate and pressure 


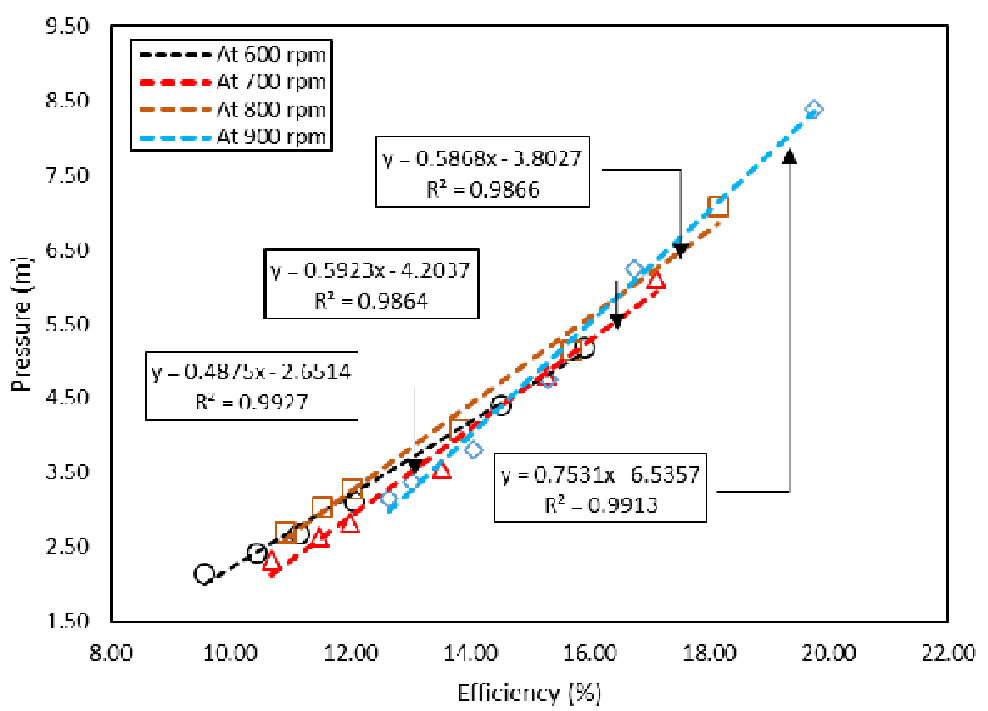

(a) The relationship between efficiency and pressure at 600 to $900 \mathrm{rpm}$.

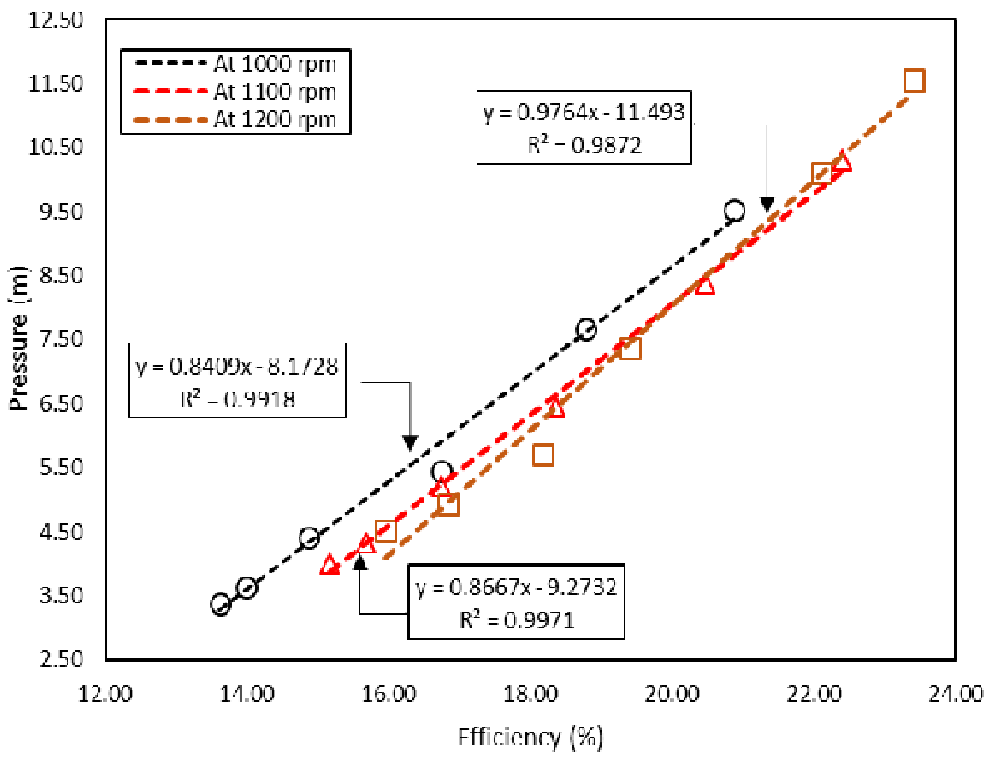

(b) The relationship between efficiency and pressure at 1000 to $1200 \mathrm{rpm}$.

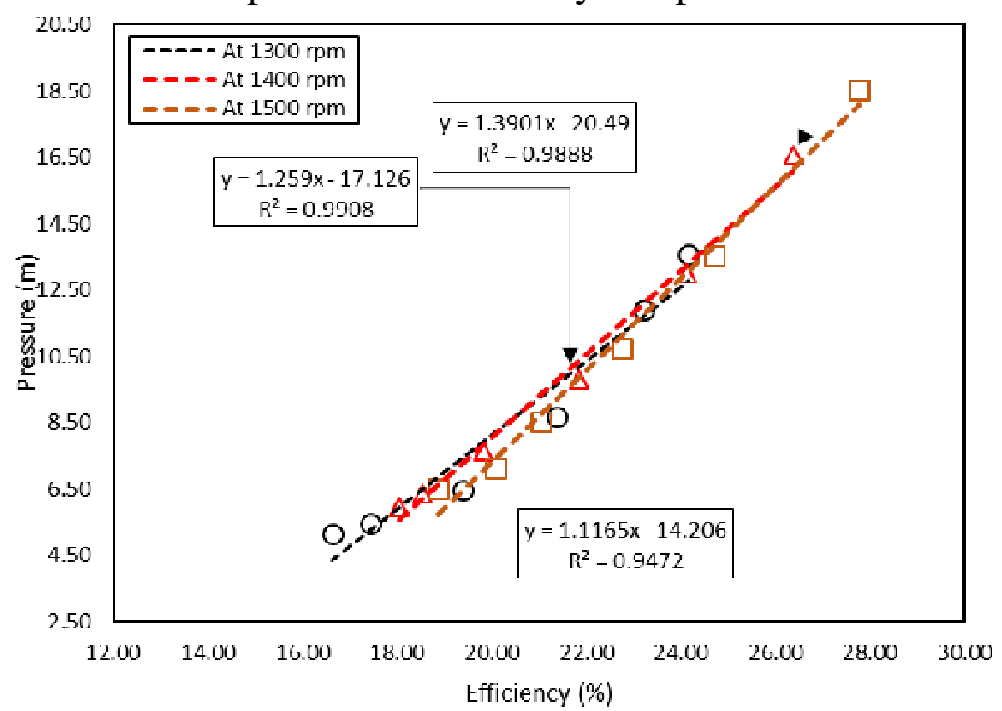

(c) The relationship between efficiency and pressure at 1300 to $1500 \mathrm{rpm}$.

Fig. 7: The relationship between efficiency and pressure 


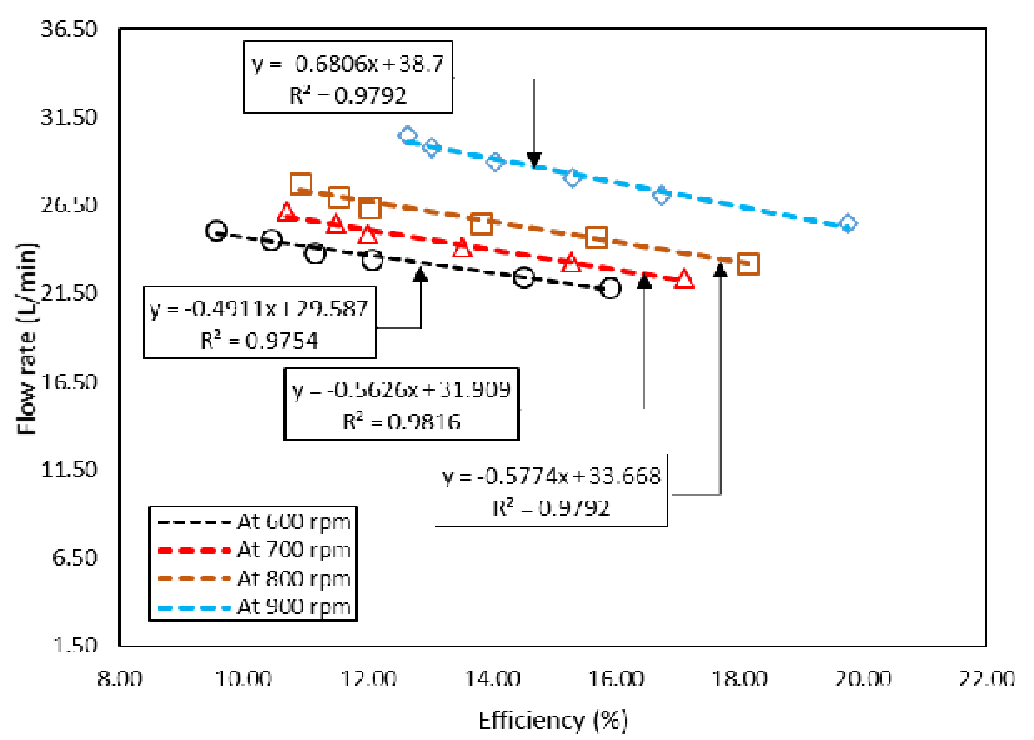

(a) The relationship between efficiency and flow rate at 600 to $900 \mathrm{rpm}$.

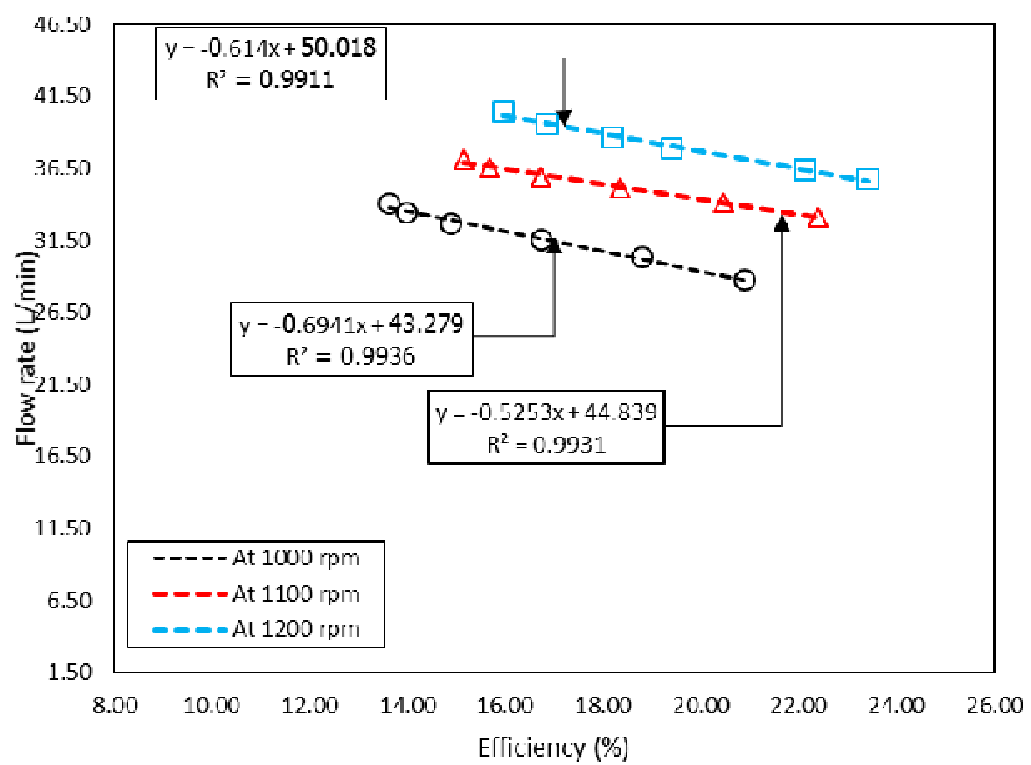

(b) The relationship between efficiency and flow rate at 1000 to $1200 \mathrm{rpm}$

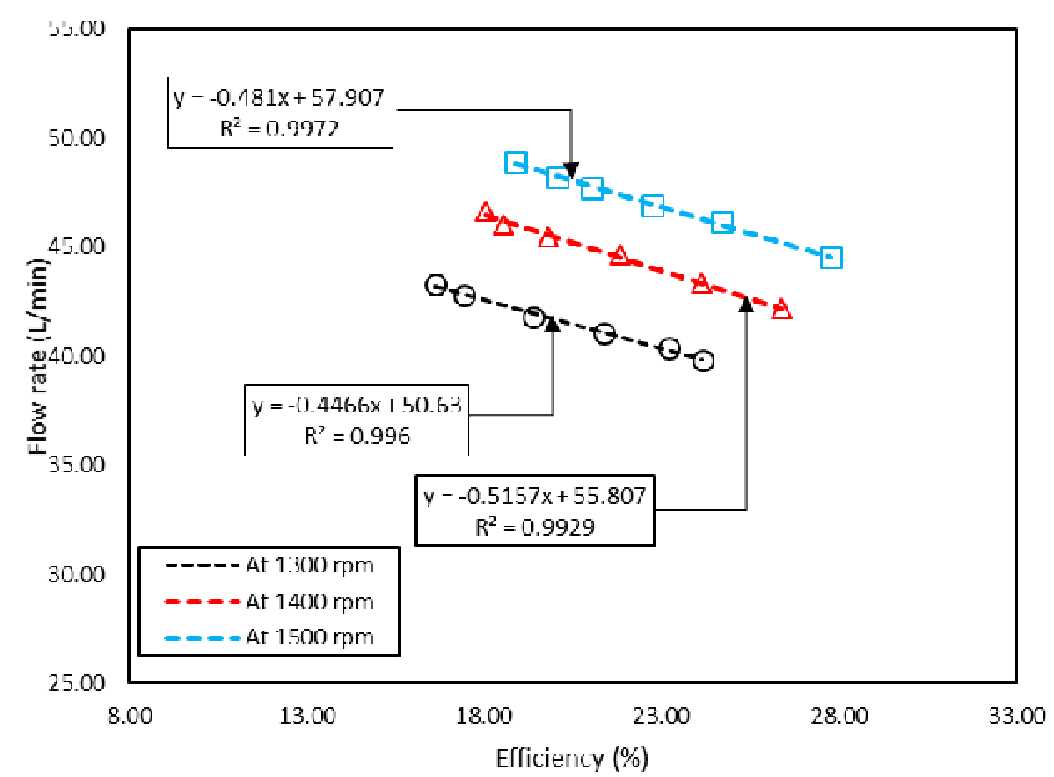

(c) The relationship between efficiency and flow rate at 1300 to $1500 \mathrm{rpm}$

Fig. 8: The relationship between efficiency and flow rate 


\section{Conclusions}

The study on the design and test a vertical scroll pump efficiency found the relationship between the pressure and the flow rate are a linear manner. If a vertical scroll pump increases the pressure, a vertical scroll pump will change the flow rate a little at the constant speed. If a vertical scroll pump increases the speed of pump, a vertical scroll pump will increase the flow rate and the pressure. At the constant speed the relationship between the efficiency of a vertical scroll pump and the pressure are a linear manner. If the pressure increase the efficiency of a vertical scroll pump will increase. In contrast, if the efficiency of a vertical scroll pump decreases the flow rate will decrease the constant speed. From the experiment the speed of a vertical scroll pump can be operated at $1500 \mathrm{rpm}$. So, if a scroll pump can be reduced the centrifugal force, a scroll pump will operate at more than $1500 \mathrm{rpm}$.

\section{Acknowledgements}

Thank you for Fluid Machinery Laboratory of Mechanical Engineering of Kasetsart University that provides the tools and equipment used in this testing.

\section{References}

[1] Kritmaitree, P. (2002). Study of Volumetric Scroll Pump for the Cold Moderator System. Ph.D. Thesis, Utsunomiya University, Japan

[2] Jeerawat Pokanit (2010). Desing and Testing A scroll Pump. Master of Engineering Thesis, the Faculty of Engineering, Kasetsart University.

[3] Sukit Litikorn (2012). Design and Testing of a Scroll Pump Efficiency. Doctor of Engineering Thesis, the Faculty of Engineering, Kasetsart University.

[4] British Standard (2005)Rotary positive displacement pumps -Performance tests for acceptance . BS EN 14343British Standards :London, United Kingdom

[5] Japan Standard Association (1990) Testing Methods for Centrifugal Pump, Mixed Flow Pump and Axial Flow Pumps. JIS B 8301 Japanese Standards Association: Tokyo, Japan 